A\&A 365, 204-213 (2001)

DOI: $10.1051 / 0004-6361: 20000180$

(c) ESO 2001

\title{
CCD photometry of distant comets II
}

\author{
S. C. Lowry ${ }^{1}$ and A. Fitzsimmons ${ }^{1}$
}

\author{
APS Division, Department of Pure and Applied Physics, The Queen's University of Belfast, Belfast BT7 1NN, UK
}

Received 6 March 2000 / Accepted 29 September 2000

\begin{abstract}
R$ band CCD photometric observations of short period Jupiter family comets in the heliocentric region of $2.11 \mathrm{AU} \leq R_{\mathrm{h}} \leq 5.63 \mathrm{AU}$ were performed using the $4.2 \mathrm{~m}$ William Herschel Telescope on La Palma in December 1998. 22 comets were targeted, including the comet-asteroid transition object 49P/Arend-Rigaux. Out of a total of ten detected comets, six were seen to display substantial outgassing (48P, 65P, 74P, 103P, 128P, and 139P), with the remaining four comets $(7 \mathrm{P}, 9 \mathrm{P}, 22 \mathrm{P}$, and $49 \mathrm{P})$ being stellar in appearance. Nuclear radius measurements and relative dust production rates in terms of $A f \rho$ were measured for these comets, along with upper limits for the remaining twelve undetected comets $(6 \mathrm{P}, 44 \mathrm{P}, 51 \mathrm{P}, 54 \mathrm{P}, 57 \mathrm{P}, 63 \mathrm{P}, 71 \mathrm{P}, 73 \mathrm{P}, 79 \mathrm{P}, 86 \mathrm{P}, 87 \mathrm{P}$, and $100 \mathrm{P})$. The inactive comets had nuclear radii in the range $1.8 \mathrm{~km} \leq r_{\mathrm{N}} \leq 4.4 \mathrm{~km}$, while upper limits for the active and undetected comets (assuming they all lay within the field of view) were between $0.6 \mathrm{~km}$ and $12.7 \mathrm{~km}$, for an assumed albedo of 0.04 . Even if one applies the previously measured maximum axis ratio of 2.6:1 and the minimum measured albedo of 0.02 to the undetected comets, their projected semi-major axes are all constrained to below $8 \mathrm{~km}$. For the active comets, photometric profiles of their dust comae were measured and are consistent with those of steady state coma models.
\end{abstract}

Key words. comets - photometry

\section{Introduction}

We present in this paper results from a long term observational program designed to help constrain the short period comet size distribution, and to study the activity levels in the region beyond $3 \mathrm{AU}$ from the sun, where the sublimation of surface volatiles is no longer dominated by $\mathrm{H}_{2} \mathrm{O}$. The majority of cometary observations are performed well within this canonical distance, resulting in a selection of techniques being utilised when characterizing the physical properties of cometary nuclei. Such techniques include that of Lamy \& Toth (1995), who use the HST to image the inner coma of comets in order to determine the nuclear flux through steady state coma modelling. A second method allows both the albedo and nuclear dimensions to be determined through simultaneous optical and infrared observations. This requires sufficiently small heliocentric distances to enable detection in the infrared and is also limited to comets that exhibit negligible activity where contamination of the nuclear signal by dust grains is minimized. Unfortunately it is rarely possible to use such techniques. In this paper we present $R$ band CCD imaging of Jupiter family comets, most of which were observed beyond $3 \mathrm{AU}$ where dust coma contamination is minimized. 49P/Arend-Rigaux was observed at 2.11 AU, well within

Send offprint requests to: S. C. Lowry, e-mail: Stephen.Lowry@qub.ac.uk the canonical $3 \mathrm{AU}$ distance, but is well known for its extremely low activity. The photometric method used includes assumptions regarding albedo and phase coefficient, which are generally taken to be 0.04 and 0.035 magnitudes/degree respectively. Provided the observations are made at small enough phase angles, then the latter assumption is relatively unimportant and the only source of error would be from unresolvable coma contamination and from the uncertainty in the assumed albedo. Detection of an inactive nucleus can be verified through observations at different heliocentric distances which was indeed the case for some of the comets discussed in the following sections. For the remaining comets, these observations may be used as a precursor for future studies.

For consistency, the structure of this paper will be similar to that of Lowry et al. (1999) (hereafter referred to as Paper I). Section 3 is again divided into subsections, describing separately the results and analysis of the undetected, inactive (unresolved), and active comets. We have reproduced some of the equations here for reasons of clarity, but where possible we refer the reader to Paper I and references therein.

\section{Observations and reduction}

$\mathrm{CCD}$ imaging of comets in the range $2.11 \mathrm{AU} \leq R_{\mathrm{h}} \leq$ $5.63 \mathrm{AU}$ was carried out on the nights of the 9th and 10th December 1998 using the Auxiliary Port camera 
Table 1. Observational geometry and $R$ filter $\log$ for all comets. $R_{\mathrm{h}}$ and $\Delta$ are the heliocentric and geocentric distances respectively. $\alpha$ is the phase angle. All images were differentially tracked at each comets apparent rate of motion

\begin{tabular}{|c|c|c|c|c|c|c|}
\hline Comet & $R_{\mathrm{h}}[\mathrm{AU}]$ & $\Delta[\mathrm{AU}]$ & $\alpha$ [deg.] & $\begin{array}{l}\text { UT date(start } \\
\text { of exposure) }\end{array}$ & Airmass & $\begin{array}{c}\text { Exposure } \\
\text { time }[\mathrm{s}]\end{array}$ \\
\hline 6P/d'Arrest & $5.63^{\dagger}$ & 5.22 & 9.49 & 11.112 & 1.497 & 600 \\
\hline 7P/Pons-Winnecke & $5.58^{\ddagger}$ & 4.61 & 1.39 & 10.218 & 1.635 & 300 \\
\hline 9P/Tempel 1 & $3.36^{\dagger}$ & 2.71 & 13.94 & 10.072 & 1.427 & 300 \\
\hline 22P/Kopff & $5.11^{\ddagger}$ & 4.14 & 2.47 & 11.092 & 1.014 & 600 \\
\hline 44P/Reinmuth 2 & $4.73^{\dagger}$ & 5.16 & 10.29 & 11.219 & 2.562 & 600 \\
\hline 48P/Johnson & $3.36^{\ddagger}$ & 2.43 & 6.41 & 9.899 & 1.354 & 300 \\
\hline 49P/Arend-Rigaux & $2.11^{\ddagger}$ & 2.44 & 23.55 & 11.272 & 1.513 & 300 \\
\hline 51P/Harrington & $5.30^{\dagger}$ & 5.73 & 9.22 & 10.263 & 1.607 & 300 \\
\hline 54P/de Vico-Swift & $5.39^{\star}$ & 5.70 & 9.68 & 11.244 & 1.577 & 600 \\
\hline 57P/duToit-Neujmin-Delporte & $5.10^{\ddagger}$ & 4.35 & 7.82 & 10.169 & 1.024 & 300 \\
\hline 63P/Wild 1 & $3.83^{\dagger}$ & 2.96 & 7.80 & 10.057 & 1.174 & 300 \\
\hline $65 \mathrm{P} / \mathrm{Gunn}$ & $4.43^{\ddagger}$ & 3.55 & 6.36 & 11.019 & 1.109 & 600 \\
\hline 71P/Clark & $4.40^{\dagger}$ & 3.60 & 8.18 & 10.148 & 1.003 & 300 \\
\hline 73P/Schwassmann-Wachmann 3 & $5.03^{\dagger}$ & 4.12 & 4.78 & 10.176 & 1.051 & 300 \\
\hline 74P/Smirnova-Chernykh & $4.24^{\dagger}$ & 3.33 & 5.73 & 9.993 & 1.035 & 300 \\
\hline 79P/du Toit-Hartley & $3.52^{\ddagger}$ & 3.78 & 14.95 & 10.806 & 1.463 & 300 \\
\hline $86 \mathrm{P} /$ Wild 3 & $4.69^{\dagger}$ & 3.78 & 5.00 & 10.158 & 1.027 & 300 \\
\hline $87 \mathrm{P} / \mathrm{Bus}$ & $4.32^{\dagger}$ & 3.39 & 4.75 & 11.009 & 1.037 & 600 \\
\hline 100P/Hartley 1 & $3.94^{\ddagger}$ & 3.91 & 14.38 & 11.244 & 1.699 & 600 \\
\hline 103P/Hartley 2 & $3.63^{\ddagger}$ & 3.93 & 14.28 & 10.235 & 1.942 & 300 \\
\hline 128P/Shoemaker-Holt 1 & $3.66^{\ddagger}$ & 3.26 & 14.97 & 11.233 & 1.087 & 600 \\
\hline 139P/Väisälä-Oterma & $3.41^{\ddagger}$ & 2.48 & 6.69 & 10.122 & 1.525 & 300 \\
\hline
\end{tabular}

$\diamond$ December 1998, † Pre-perihelion, $\ddagger$ Post-perihelion, $\star$ At aphelion.

of the $4.2 \mathrm{~m}$ William Herschel Telescope on the Island of La Palma. A TEK $1024 \times 1024$ pixel CCD was used at the Auxiliary Port focus resulting in an image scale of 0.11 arcsec per pixel. The observing conditions were photometric over both nights. Table 1 lists the observational circumstances for each of the 22 targeted comets. All cometary images were obtained with the telescope tracking at their respective apparent rates of motion, therefore taking multiple images of the same field for the purposes of object identification was unnecessary as the only untrailed object in the frame would be the comet. Additional search and detection criteria included astrometry of field stars to enable inspection of the exact predicted position of the comet. The exposure times were limited to either 300 or $600 \mathrm{~s}$ and were taken using a Harris $R$ band filter ( $F W H M \sim 150 \mathrm{~nm}$, peak efficiency at $\sim 594 \mathrm{~nm})$. A log of all cometary observations is also given in Table 1. Each CCD image was bias subtracted and subsequently flat-fielded using twilight sky exposures. To calibrate the images a number of exposures of the standard fields SA96 and SA101 (Landolt 1992) were taken throughout each night. As only $R$ band imaging was performed the $(V-R)$ colour index of the $\operatorname{sun}^{1}$ was assumed for each comet in order to convert from instrumental to apparent magnitudes. The errors introduced from this assumption were found to be negligible for true cometary $(V-R)$ values $\leq 0.8$. Image processing and photometry was performed with the IRAF/CCDRED (Massey 1997),

\footnotetext{
${ }^{1}(V-R)_{\odot}=0.36($ Meech et al. 1995).
}

IRAF/APPHOT (Davis 1989) and IRAF/DAOPHOT (Davis 1994) packages on the Northern Ireland STARLINK node.

\section{Results and analysis}

\subsection{Undetected comets}

6P/d'Arrest, 44P/Reinmuth 2, 51P/Harrington, 54P/de Vico-Swift, 57P/duToit-Neujmin-Delporte, 63P/Wild 1, 71P/Clark, 73P/Schwassmann-Wachmann 3, 79P/du Toit-Hartley, 86P/Wild 3, 87P/Bus, 100P/Hartley 1

To calculate the limiting magnitude of each cometary image the standard deviation of the background noise at the expected position of the comet was measured, and used along with the following expression for faint objects:

$S / N=\frac{I . t}{\sqrt{\pi r^{2}} \cdot \sigma}$

where $\pi r^{2}$ is the number of pixels within the aperture. Here $r=1.6 \times F W H M$ which is the optimal radius for a Gaussian profile (Pritchet \& Kline 1981). The full width half maximum was calculated using the round ends of background stellar trails. $I$ is the total measured intensity $[\mathrm{ADUs} / \mathrm{s}]$ of the object within the aperture, $t$ is the exposure time and $\sigma$ is the standard deviation of the background noise. If we assume $S / N \geq 3$ for the object to be detectable we can calculate an upper limit for $I$ which can then be transformed to a limiting magnitude. The $3 \sigma$ limiting magnitudes derived for each cometary frame 
Table 2. Limiting $R$ band magnitudes, nuclear radius upper limits (based on assumed albedos of 0.04 and 0.02 ), and $A f \rho$ upper limits for the undetected comets

\begin{tabular}{lcccc}
\hline Comet & $\begin{array}{c}\text { Limiting } R \text { band } \\
\text { magnitude }\end{array}$ & $\begin{array}{c}r_{\mathrm{N}}^{\star}[\mathrm{km}] \\
A_{\mathrm{R}}=0.04\end{array}$ & $\begin{array}{c}r_{\mathrm{N}}^{\star}[\mathrm{km}] \\
A_{\mathrm{R}}=0.02\end{array}$ & $\begin{array}{c}\text { Af } \rho \text { upper } \\
\text { limit }^{\star}[\mathrm{cm}]\end{array}$ \\
\hline 6P/d'Arrest & 23.2 & $\leq 2.1$ & $\leq 3.0$ & $\leq 14.2$ \\
44P/Reinmuth 2 & 23.6 & $\leq 1.4$ & $\leq 2.0$ & $\leq 2.3$ \\
51P/Harrington & 23.5 & $\leq 1.9^{\dagger}$ & $\leq 2.6^{\dagger}$ & $\leq 5.9$ \\
54P/de Vico-Swift & 23.2 & $\leq 2.1^{\dagger}$ & $\leq 3.0^{\dagger}$ & $\leq 6.1$ \\
57P/duToit-Neujmin-Delporte & 24.0 & $\leq 1.1$ & $\leq 1.5$ & $\leq 2.9$ \\
63P/Wild 1 & 23.8 & $\leq 0.6$ & $\leq 0.8$ & $\leq 1.1$ \\
71P/Clark & 23.7 & $\leq 0.9$ & $\leq 1.2$ & $\leq 2.4$ \\
73P/Schwassmann-Wachmann $3^{\diamond}$ & 24.2 & $\leq 0.9^{\dagger}$ & $\leq 1.2^{\dagger}$ & $\leq 2.7$ \\
79P/du Toit-Hartley & 22.4 & $\leq 1.5$ & $\leq 2.1$ & $\leq 1.5$ \\
86P/Wild 3 & 23.9 & $\leq 0.9$ & $\leq 1.2$ & $\leq 2.2$ \\
87P/Bus & 24.4 & $\leq 0.6^{\dagger}$ & $\leq 0.8^{\dagger}$ & $\leq 0.8$ \\
100P/Hartley 1 & 23.2 & $\leq 1.2^{\dagger}$ & $\leq 1.7^{\dagger}$ & $\leq 1.2$ \\
\hline
\end{tabular}

$\star 3 \sigma$ upper limits, $A_{\mathrm{R}}$ is the assumed nuclear albedo, $r_{\mathrm{N}}$ is the nuclear radius, $\dagger$ The radius upper limits for these comets are less reliable and should be treated with caution (see Sect. 3.1), $\diamond$ Note that the nuclei of 51P/Harrington \& 73P/SchwassmannWachmann 3 have split within a few years prior to our observations (Scotti \& Jedicke 1994; Boehnhardt \& Käufl 1995).

are listed in Table 2. Taking these limiting magnitudes as the upper limit to the comet brightness, upper limits to their nuclear radii were derived by substituting them for $m_{\mathrm{R}}$ in the following expression of Russell (1916):

$A_{\mathrm{R}} C=\frac{2.2510^{22} R_{\mathrm{h}}^{2} \pi \Delta^{2} 10^{0.4\left(m_{\odot}-m_{\mathrm{R}}\right)}}{10^{-0.4 \alpha \beta}}$

where $A_{\mathrm{R}}$ is the geometric albedo in the $R$ filter, $C\left[\mathrm{~m}^{2}\right]$ is the geometrical cross-section of the nucleus, $R_{\mathrm{h}}[\mathrm{AU}]$ and $\Delta[\mathrm{AU}]$ are the heliocentric and geocentric distances respectively, $\alpha$ and $\beta$ are the phase angle and phase coefficient respectively. $m_{\odot}$ and $m_{\mathrm{R}}$ are the apparent $R$ magnitudes of the sun and comet respectively. With the exception of 49P/Arend-Rigaux, for which an albedo and phase coefficient have been measured, empirically derived values for the albedo of 0.04 (see Fig. 6 of Fitzsimmons et al. 1994 and references therein) and phase coefficient of $0.035 \pm 0.005$ (Sekanina 1974; Meech \& Jewitt 1987; Jewitt \& Meech 1987a; Jewitt \& Meech 1988; Jewitt \& Luu 1989) are assumed. Additional uncertainties introduced through these assumptions are discussed further in Sect. 4.2.

The orbits of numbered short period comets are generally well established, but in order for the upper limits derived above to be reliable, some assessment should be made of the likelihood that the comets are actually outside our field of view, an outcome that would render any derived radius upper limit meaningless. For each comet listed in Table 2, the area searched was 2.76 square arcminutes. For all but a few of the comets listed in Table 2, astrometric measurements have been performed within 0-3 years prior to our observations, and in some cases 0-2 years afterwards. For those comets with astrometric data taken before and after our observations $(6 \mathrm{P} / \mathrm{d}$ 'Arrest, $44 \mathrm{P} /$ Reinmuth 2 , and $63 \mathrm{P} /$ Wild 1 ), the expected uncertainty in position was negligible. For those comets possessing astrometric data taken $0-3$ years prior to our observations $(57 \mathrm{P} / \mathrm{du}$ Toit-Neujmin-Delporte, 71P/Clark, and
86P/Wild 3), we compared the expected change in orbital period caused by non-gravitational forces acting on the comet $^{2}$ between the dates of the most recent astrometric observations and our observations, with the change in orbital period that would be required to remove the comet from our field of view. In every case, any advance or delay of the comet due to previously measured non-gravitational forces was insufficient to remove the comet from the frame. Unless a major gravitational perturbation or outburst has occurred, the radius upper limits of these comets may be regarded as firm. The radius upper limits of $87 \mathrm{P} / \mathrm{Bus}$, 100P/Hartley 1, 54P/de Vico-Swift, 73P/SchwassmannWachmann 3 , and $51 \mathrm{P} /$ Harrington are less reliable as it cannot be confirmed that they fell within our search area. This was due to either large non-gravitational forces, the lack of recent astrometric data, or that the nuclei have been known to split within a few years of our observations (as in the case of 51P/Harrington and 73P/SchwassmannWachmann 3, Scotti \& Jedicke 1994; Boehnhardt \& Käufl 1995). However, they have been included in the event that future astrometric data may provide a positive confirmation of their suspected positions.

To calculate an upper limit to the relative dust production rate the quantity $A f \rho$ is used (A'Hearn et al. 1984). This aperture independent quantity is roughly proportional to the dust production rate of a comet assuming equal size distributions of particles in the coma and can be determined from the observations using:

$A f \rho[\mathrm{cm}]=\frac{\left(2 \Delta R_{\mathrm{h}}\right)^{2} F_{\text {comet }}}{\rho F_{\odot}}$

where $A$ is the geometric albedo of the cometary dust grains, the filling factor $f$ is the total cross-section of the grains in the field of view divided by the area of the field of view, and $\rho[\mathrm{cm}]$ is the radius of the field of view. $R_{\mathrm{h}}[\mathrm{AU}]$

\footnotetext{
${ }^{2}$ See Eq. (5.6) of Festou et al. (1993).
} 
(a) 7P/Pons-Winnecke

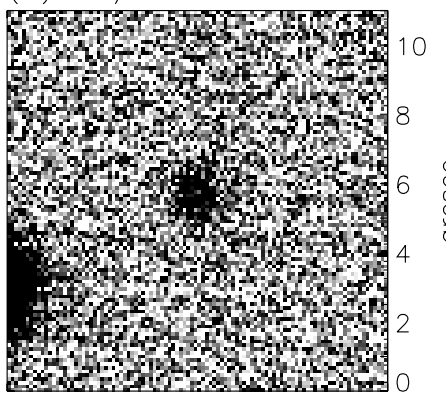

(d) 48P/Johnson

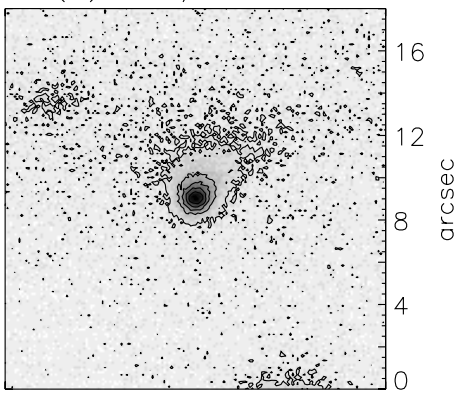

(g) $103 \mathrm{P} /$ Hartley 2

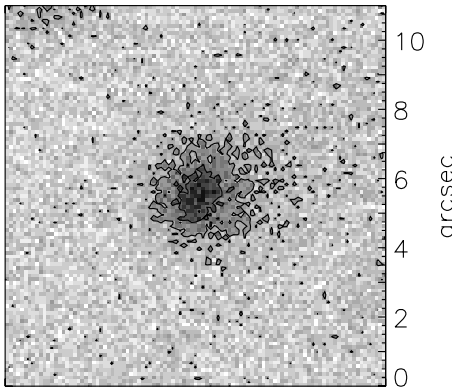

(b) $9 \mathrm{P} /$ Tempel 1

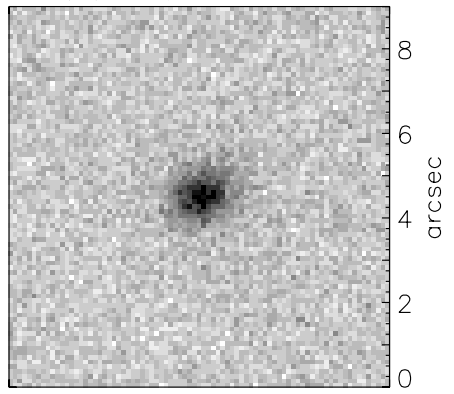

(e) 65 P/Gunn

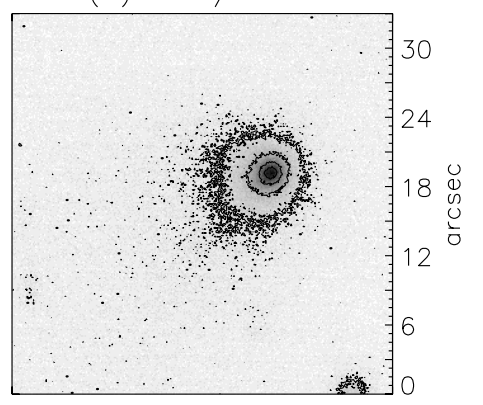

(h) $128 \mathrm{P} /$ Shoemaker-Holt 1

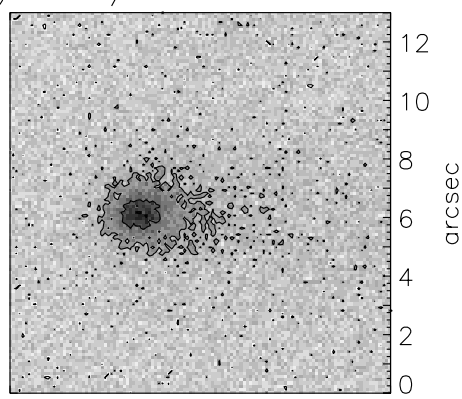

(c) $22 \mathrm{P} / \mathrm{Kopff}$
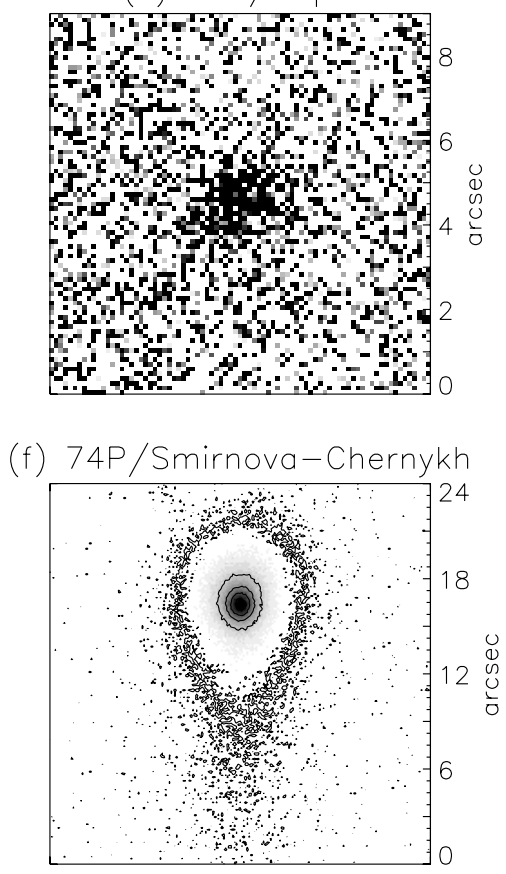

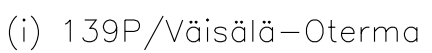

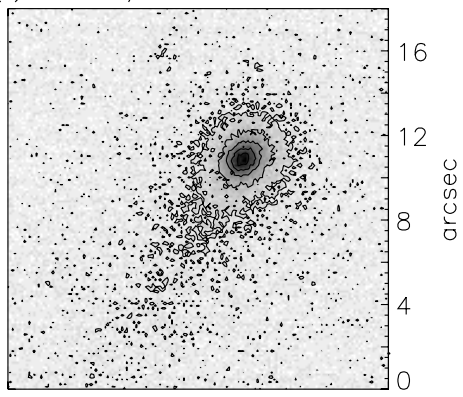

Fig. 1. $R$ band CCD images of all the detected comets, with the exception of 49P/Arend-Rigaux (see Fig. 3b of Sect. 4.1). Each of the active comets has overplotted contours to enhance some of the dust comae features

and $\Delta[\mathrm{cm}]$ are the heliocentric and geocentric distances respectively and $F_{\text {comet }} / F_{\odot}$ is the ratio of the cometary and solar flux at the observed wavelength. $F_{\text {comet }}$ is derived from the limiting magnitudes and $\rho$ is equal to $r$, as defined in Eq. (1), multiplied by the pixel scale [cm/pixel] of each cometary frame. The values obtained are also listed in Table 2.

\subsection{Unresolved comets}

7P/Pons-Winnecke, 9P/Tempel 1, 22P/Kopff, $49 P /$ Arend-Rigaux

Figures $1 \mathrm{a}-\mathrm{c}$ and $3 \mathrm{~b}$ are the CCD images that were taken of these four comets. Comparison of their profiles with the scaled profiles of the round ends of trailed background stars showed that these comets appeared as unresolved point sources. An example of this is shown in Fig. 3a. Due to the differential tracking of these objects it was not possible to accurately reconstruct the complete stellar background Point Spread Function (PSF), therefore the method of subtracting the average scaled PSF from the comet to search for signs of a coma was not applied to any of the observations discussed in this paper.

Apparent $R$ band magnitudes were measured and Eq. (2) was used to calculate the nuclear radius measurements which are listed in Table 3. To ascertain the significance of any possible coma contribution to the $R$ band magnitude and hence the nuclear radius measurements, the expression of Jewitt \& Danielson (1984) is used along with the method described in Sect. 3.2. of Paper I. For $7 \mathrm{P} /$ Pons-Winnecke $m_{\text {coma }} \geq 21.87 \pm 0.03$, therefore comparison of this value with its $R$ band magnitude of $22.2 \pm 0.1$ suggests that an unresolved coma could dominate the observed flux. If this were the case it would render its nucleus radius measurement an upper limit. The same is true for $22 \mathrm{P} / \mathrm{Kopff}$. In contrast, the $R$ band magnitudes of $9 \mathrm{P} /$ Tempel 1 and 49P/Arend-Rigaux are very much greater than their calculated $m_{\text {coma }}$ values. Therefore, the nucleus radius measurements of $2.4 \pm 0.1 \mathrm{~km}$ and $4.4 \pm 0.1 \mathrm{~km}$ for $9 \mathrm{P} /$ Tempel 1 and $49 \mathrm{P} /$ Arend-Rigaux respectively are unlikely to be affected 
Table 3. $R$ band magnitudes, nuclear radius measurements and upper limits (based on assumed albedos of 0.04 and 0.02 ), and Af $\rho$ values and upper limits for the detected comets. Note the different value of albedo for 49P/Arend-Rigaux

\begin{tabular}{|c|c|c|c|c|c|c|}
\hline Comet & $\begin{array}{c}R \text { band } \\
\text { magnitude }\end{array}$ & $\begin{array}{c}r_{\mathrm{N}}[\mathrm{km}] \\
A_{\mathrm{R}}=0.04\end{array}$ & $\begin{array}{c}r_{\mathrm{N}}[\mathrm{km}] \\
A_{\mathrm{R}}=0.02\end{array}$ & $m_{\text {coma }}$ & $\begin{array}{c}A f \rho \\
\text { value }[\mathrm{cm}]\end{array}$ & $\begin{array}{c}f \rho \text { radius } \\
\text { [arcsec] }\end{array}$ \\
\hline \multicolumn{7}{|c|}{ UNRESOLVED COMETS } \\
\hline 7P/Pons-Winnecke & $22.2 \pm 0.1$ & $2.6 \pm 0.1$ & - & $21.87 \pm 0.03$ & $\leq 20.0^{\star}$ & 2.0 \\
\hline 9P/Tempel 1 & $20.5 \pm 0.1$ & $2.4 \pm 0.1$ & - & $21.60 \pm 0.03$ & $\leq 11.2^{\star}$ & 3.4 \\
\hline $22 \mathrm{P} / \mathrm{Kopff}$ & $22.6 \pm 0.1$ & $1.8 \pm 0.1$ & - & $21.67 \pm 0.02$ & $\leq 8.9^{\star}$ & 2.2 \\
\hline 49P/Arend-Rigaux & $18.60 \pm 0.03$ & $4.4 \pm 0.1^{\ddagger}$ & - & $22.47 \pm 0.03$ & $\leq 17.1^{\star}$ & 3.7 \\
\hline \multicolumn{7}{|l|}{ ACTIVE COMETS } \\
\hline 48P/Johnson & $18.55 \pm 0.04$ & $\leq 3.5^{\star}$ & $\leq 4.8^{\star}$ & - & $28.1 \pm 1.1$ & 3.8 \\
\hline $65 \mathrm{P} /$ Gunn & $17.74 \pm 0.04$ & $\leq 8.8^{\star}$ & $\leq 12.2^{\star}$ & - & $133.4 \pm 4.7$ & 2.6 \\
\hline 74P/Smirnova-Chernykh & $16.78 \pm 0.04$ & $\leq 12.7^{\star}$ & $\leq 18.0^{\star}$ & - & $298.9 \pm 11.3$ & 2.7 \\
\hline 103P/Hartley 2 & $19.01 \pm 0.04$ & $\leq 5.9^{\star}$ & $\leq 8.4^{\star}$ & - & $49.3 \pm 4.8$ & 2.3 \\
\hline 128P/Shoemaker-Holt 1 & $19.91 \pm 0.05$ & $\leq 4.0^{\star}$ & $\leq 5.6^{\star}$ & - & $19.3 \pm 0.7$ & 2.8 \\
\hline 139P/Väisälä-Oterma & $18.16 \pm 0.04$ & $\leq 4.6^{\star}$ & $\leq 6.4^{\star}$ & - & $47.7 \pm 1.8$ & 4.1 \\
\hline
\end{tabular}

$\star 3 \sigma$ upper limits, $\dagger$ Radius of photometric aperture used to determine $A f \rho, A_{\mathrm{R}}$ is the assumed nuclear albedo, $r_{\mathrm{N}}$ is the nuclear radius, $\ddagger A_{\mathrm{R}}=0.03$ (Millis et al. 1988).

by any coma contamination. Taking 49P/Arend-Rigaux as an example, the maximum contribution of an unresolved $1 / r$ coma to the total flux is $\sim 3 \%$.

Af $\rho$ upper limits were also calculated for these comets using an arbitrary value for $\rho$ of $6600 \mathrm{~km}$. Table 3 lists the upper limits along with the photometric radii used in their derivation.

\subsection{Active comets}

48P/Johnson, 65P/Gunn, 74P/Smirnova-Chernykh, 103P/Hartley 2, 128P/Shoemaker-Holt 1, 139P/VäisäläOterma

Images of these comets can be seen in Figs. 1d-i which have overplotted contours to enhance some of the coma morphology. They show the extent of the dust comae, particularly in the case of $74 \mathrm{P} /$ Smirnova-Chernykh and $65 \mathrm{P} /$ Gunn whose activity dwarfs that of the other comets.

$R$ band magnitudes and Af $\rho$ values were measured and are listed in Table 3. The same arbitrary value for $\rho$ of $6600 \mathrm{~km}$ was used for the $A f \rho$ values of each comet. For the derived $A f \rho$ values to be useful it should be possible to describe their comae using a steady state coma model. Therefore in order to conveniently compare their comae with such a model their surface brightness profiles were calculated. This was done by measuring the cometary flux through a series of circular annuli $2-5$ pixels wide. The resulting plots of surface brightness $\left[\mathrm{mag} / \mathrm{arcsec}^{2}\right]$ vs. $\log (r)$ [arcsec] are shown in Fig. 2. Overplotted on each graph are two diagonal lines with gradients of -1.5 and -1 which represent steady state coma models with and without the effects of radiation pressure respectively (Jewitt \& Meech 1987b). After inspection of each surface brightness profile it is apparent that all the comae can be represented by such a model within the photometric uncertainties, hence Eq. (3) can be applied.

To derive the nuclear radius upper limits, the flux was measured through apertures whose radii were chosen such that any coma contribution would be minimal and yet would easily include all flux from the nucleus. This radius was dependent on the FWHM of the background stars, and was taken to be the point where the background PSF drops to $\sim 2 \%$ of its peak intensity. $R$ band magnitudes were thus measured for $48 \mathrm{P} /$ Johnson, $65 \mathrm{P} / \mathrm{Gunn}, 74 \mathrm{P} /$ SmirnovaChernykh, 103P/Hartley 2, 128P/Shoemaker-Holt 1, and $139 \mathrm{P} /$ Väisälä-Oterma through apertures of radii $2.1^{\prime \prime}$, $2.5^{\prime \prime}, 2.6^{\prime \prime}, 2.2^{\prime \prime}, 3.1^{\prime \prime}$, and $2.5^{\prime \prime}$ respectively and substituted into Eq. (2). The resulting upper limits are given in Table 3. Unfortunately, due to the differential tracking, the PSF subtraction technique discussed in Sect. 3.3.1 of Paper I cannot be used to refine these upper limits because of the inability to accurately reconstruct the background PSF.

\section{Discussion}

\subsection{P/Arend-Rigaux}

Observed at a heliocentric distance of $2.11 \mathrm{AU}$, this comet is virtually inactive but extremely bright with an $R$ magnitude of $18.60 \pm 0.03$, even brighter than some of the active comets such as $103 \mathrm{P} /$ Hartley 2 and $128 \mathrm{P} /$ Shoemaker-Holt 1 (albeit observed at larger $R_{\mathrm{h}}$ ). Figure 3a compares the brightness profile of $49 \mathrm{P} /$ ArendRigaux with that of a scaled background star, illustrating its stellar appearance. Their profiles are indistinguishable in almost all directions. Within the $R$ band CCD image seen in Fig. $3 \mathrm{~b}$ an extremely faint narrow jet can be seen emanating from the comet towards the top-right corner of the image. The position angle of this jet is $\sim 60^{\circ}$, i.e. exactly along the anti-solar direction as projected onto the plane of the sky. One may immediately attribute this feature to weak $\mathrm{H}_{2} \mathrm{O}^{+}$ emissions from an ion tail (which would certainly show up on an $R$ band image). However, we stress that no 
(a) 48P/Johnson

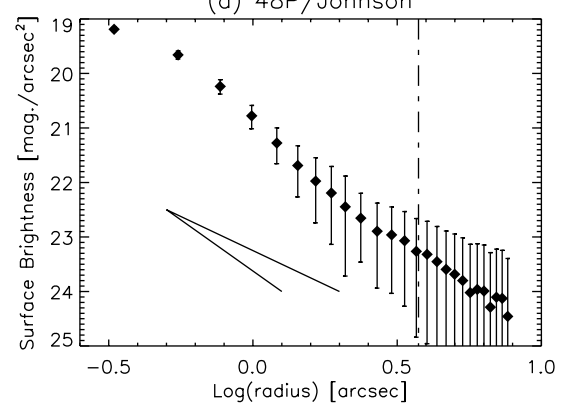

(d) $103 \mathrm{P} /$ Hartley 2

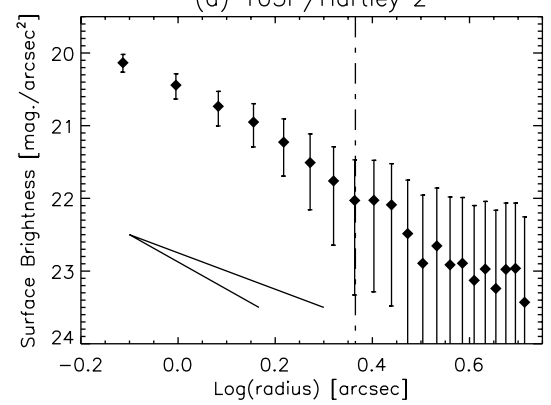

(b) $65 \mathrm{P} /$ Gunn

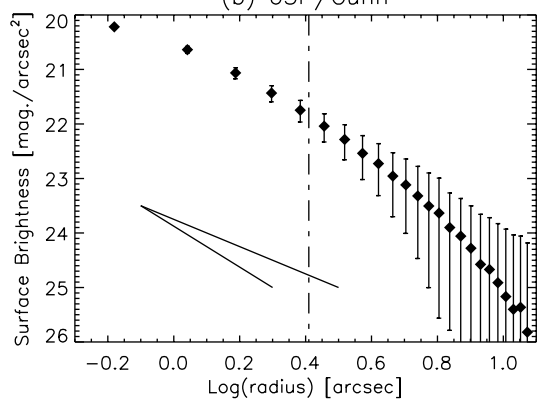

(e) $128 \mathrm{P} /$ Shoemaker-Holt 1

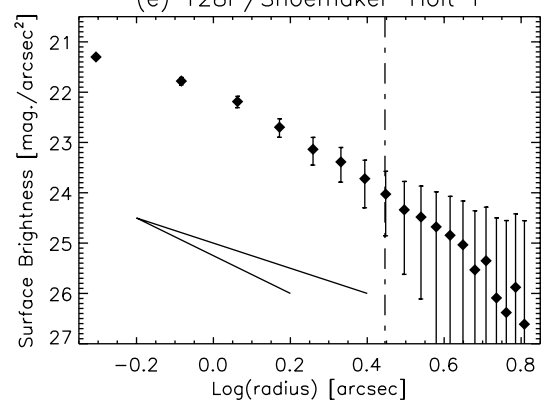

(c) $74 \mathrm{P} /$ Smirnova-Chernykh

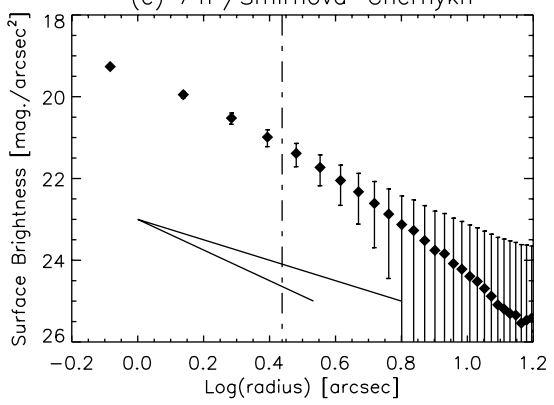

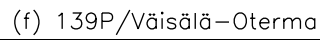

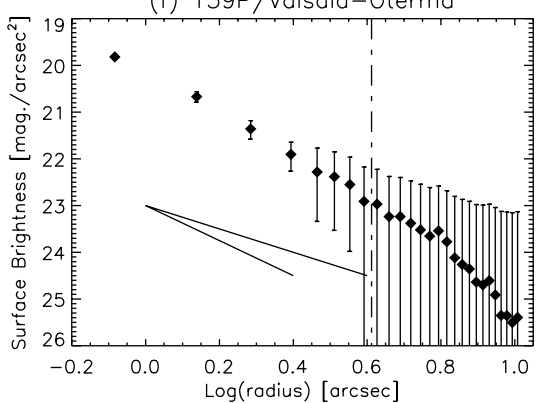

Fig. 2. Azimuthally averaged surface brightness profiles of the active comets. The two diagonal lines on each graph, with gradients of -1.5 and -1 , represent steady state coma models with and without the effects of radiation pressure respectively. The vertical dashed lines are the Af $\rho$ radii

(a)

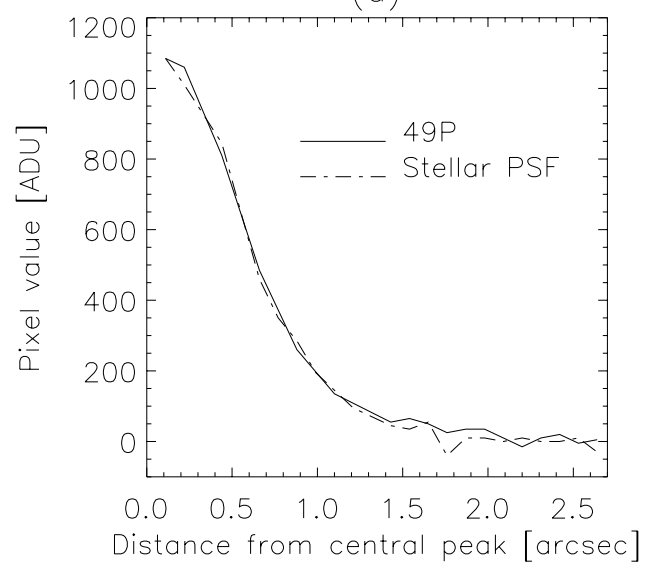

(b)

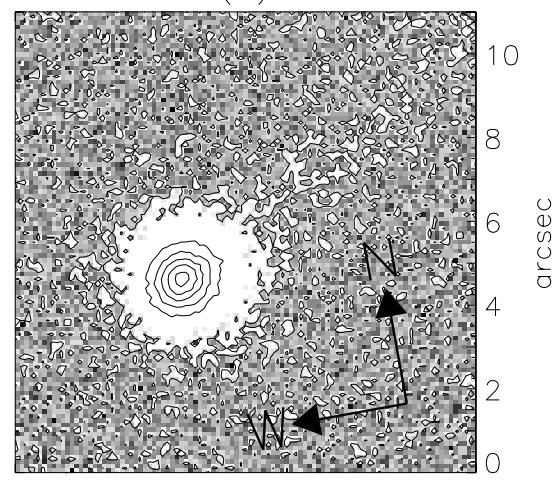

Fig. 3. a) compares the brightness profile of $49 \mathrm{P} /$ Arend-Riguax with that of a scaled background star, illustrating the stellar appearance of the comet. $\mathbf{b}$ ) is an $R$ band CCD image with overplotted contours to enhance the faint narrow dust-jet extending towards the top-right corner of the image

resolvable coma was observed despite the good seeing and relatively small heliocentric and geocentric distances. Earth was near the orbital plane of $49 \mathrm{P} /$ Arend-Rigaux at the time of these observations, with an "Earth-cometorbital plane" angle of $4.9^{\circ}$. Therefore the presence of a dust tail caused by projection effects increasing the observed column density is plausible. Another possibilty is that this narrow feature is a dust-jet emanating from an extremely small active portion of the nucleus surface, perhaps due to occasional pressure buildups near areas of thin mantle resulting in temporary outbursts in activity. Ultimately, these observations demonstrate that this comet remains active at small heliocentric distances, although the activity is barely detectable. However, more observations are desirable in order to monitor any possible transition to an asteroid-like state. Such a transition has been strongly suggested based on extensive previous investigations.

49P/Arend-Rigaux is one of the few comets for which an albedo and phase coefficient have been measured, with the values being 0.03 (Millis et al. 1988) and $0.035 \mathrm{mag} /$ degree (Sekanina 1974) respectively. Additionally, 49P/Arend-Rigaux is one of the few cometary nuclei for which the phase law has been measured beyond $20^{\circ}$, which is fortunate given that our observations were obtained at a phase angle of $23.55^{\circ}$. A nucleus radius 


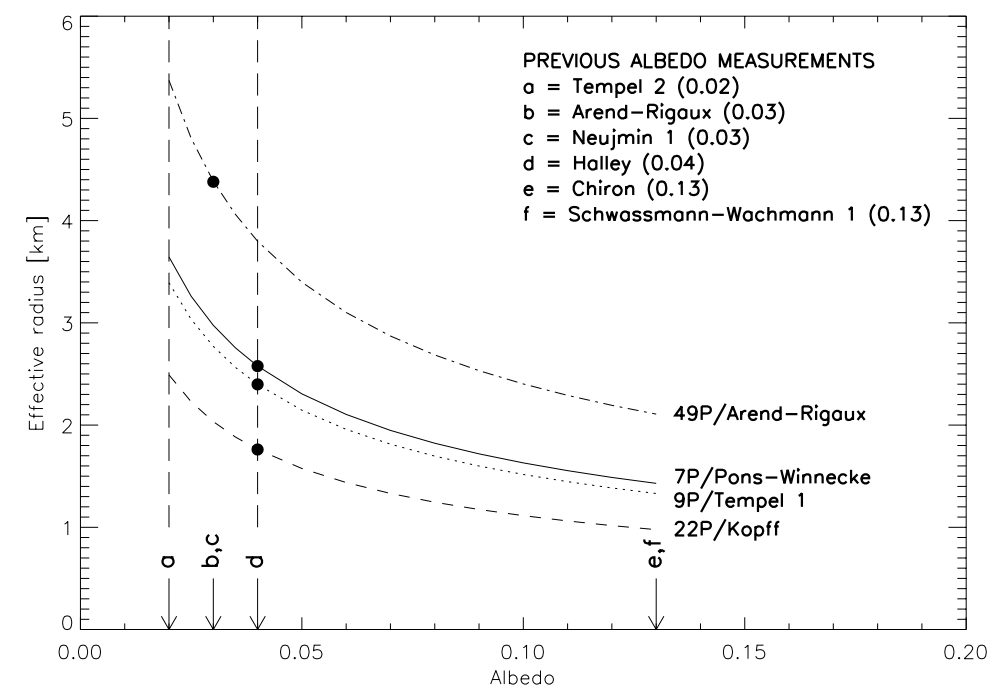

Fig. 4. Nuclear radius as a function of the assumed albedo for the unresolved comets of Sect. 3.2. The filled circles highlight the position of each of the unresolved comets on their respective radius/albedo curves for our adopted albedos. 49P/Arend-Rigaux is included for comparison even though an albedo of 0.03 has been confirmed. The region between the two vertical dashed lines represents the optimal range of assumed albedos, as within this region the bulk of measured albedos reside. References: [a] A'Hearn et al. (1989), [b] Millis et al. (1988), [c] Campins et al. (1987), [d] Keller et al. (1987), [e] Bus et al. (1989), [f] Cruikshank \& Brown (1983)

of $4.4 \pm 0.1 \mathrm{~km}$ was derived based on its $R$ magnitude of $18.60 \pm 0.03$ (measured through a 5.3 arcsec aperture) and the previously measured values of albedo and phase coefficient. This is consistent with previous measurements of $4.8 \pm 0.4 \mathrm{~km}$ (Tokunaga \& Hanner 1985), $5.1 \pm 1.1 \mathrm{~km}$ (Brooke \& Knacke 1986), and 4.5 $5_{-0.5}^{+0.2} \mathrm{~km}$ (Birkett et al. 1987). Also, by taking into account the observed nuclear lightcurve, Veeder et al. (1987) found that their observations were consistent with that of a triaxial ellipsoid with radii $3.8,3.8$, and $6.8 \mathrm{~km}$. It should be noted that as the nucleus is non-spherical and rotating, our nucleus radius measurement is valid only for the particular viewing angle at the time of our observations.

\subsection{Nuclear radius measurements}

The nuclear radius measurements derived in this paper range from $1.8 \mathrm{~km} \leq r_{\mathrm{N}} \leq 4.4 \mathrm{~km}$, and the upper limits derived span the range $0.6 \mathrm{~km} \leq r_{\mathrm{N}} \leq 12.7 \mathrm{~km}$ (for an assumed albedo of 0.04). We note that all quoted errors in Tables 2 and 3 are due to the photometry alone. Additional sources of uncertainty shall now be discussed.

The albedos of cometary nuclei are generally assumed to be 0.04 , but nuclear albedos measured to date range from $0.02 \rightarrow 0.13$ (see Fig. 4). Hence, for the unresolved comets, we have illustrated in Fig. 4 how the derived radius values vary as a function of the assumed albedo. The region between the two vertical dashed lines represents the optimal range of assumed albedos, as within this region the bulk of measured albedos reside. The filled circles highlight the position of each of the unresolved comets on their respective radius/albedo curves for our adopted albedos. Note that 49P/Arend-Rigaux has a measured albedo of 0.03. For the undetected and active comets, for which upper limits have been measured, we have derived upper limits based on the minimum measured albedo of 0.02 . This subsequently leads to slightly larger upper limits which are given in Col. 4 of Tables 2 and 3. With regards to the uncertainty in the assumed phase coefficient of $0.005 \mathrm{mag} /$ degree (as introduced in Sect. 3.1), an additional uncertainty of no greater than $0.1 \mathrm{~km}$ for the derived nuclear radii values and upper limits of the unresolved and undetected comets is incurred. For the active comets a slightly larger additional uncertainty of $\leq 0.2 \mathrm{~km}$ is required.

Table 4 lists those comets with previous nuclear radius measurements and upper limits derived from photometry, and lower limits derived from the amount of active area required to produce the measured $\mathrm{OH}$ production rates listed in A'Hearn et al. (1995). With the exception of $9 \mathrm{P} /$ Tempel 1 our values and upper limits are consistent with the previous measurements. $6 \mathrm{P} / \mathrm{d}$ 'Arrest was observed by Fay \& Wisniewski (1978) and a mean apparent $V$ band magnitude of 11.60 was obtained. Unfortunately the coma contribution to the observed flux was unknown which resulted in a range of possible values for $N$, where $N$ is the ratio of the constant nuclear flux to the total observed flux. The most probable range of values for $N$ was $0.1 \leq N \leq 0.7$. Assuming an albedo of 0.04 , we find that their mean $V$ band apparent magnitude of 11.60 will lead to a range of nuclear radius values of $1.3 \leq r_{\mathrm{N}} \leq 3.4 \mathrm{~km}$ when one considers the possible range of $N$. Our derived upper limit of $\leq 2.1 \mathrm{~km}$ (also assuming $\left.A_{\mathrm{R}}=0.04\right)$ is therefore consistent with the photometry of Fay and Wisniewski. Weissman et al. (1999) also observed 9P/Tempel 1 in December 1998 and was found 
Table 4. Comparison of nuclear radius measurements with previous values

\begin{tabular}{lccc}
\hline Comet & Nuclear radius $[\mathrm{km}]$ & Previous values $^{\star}[\mathrm{km}]$ & Ref. \\
\hline 6P/d'Arrest & $\leq 2.1$ & $1.3-3.4^{\diamond}, 2.7$ & 1,2 \\
& & $\geq 0.4$ & 3 \\
9P/Tempel 1 & $2.4 \pm 0.1$ & $3.9 \times 2.9,3.9 \times 2.8$ & 4,5 \\
22P/Kopff & $1.8 \pm 0.1$ & $1.65-1.92$ & 6 \\
& & $\geq 1.0$ & 3 \\
65P/Gunn & $\leq 8.8$ & $\geq 2.2$ & 3 \\
73P/Schwassmann-Wachmann 3 & $\leq 0.9$ & $\leq 1.1^{\dagger}$ & 7 \\
79P/du Toit-Hartley & $\leq 1.5$ & $1.4 \pm 0.3$ & 8 \\
86P/Wild 3 & $\leq 0.9$ & $1.1 \pm 0.2$ & 8 \\
103P/Hartley 2 & $\leq 5.9$ & $\geq 0.6$ & 3 \\
\hline
\end{tabular}

$\star$ The lower limits are based on the $\mathrm{OH}$ production rates from A'Hearn et al. (1995), see text. $\dagger$ This measurement was made before the comet split into several pieces in 1995 (Boehnhardt \& Käufl 1995). $\diamond$ This range of values was derived using a mean apparent $V$ band magnitude of 11.60 (Fay \& Wisniewski 1978), an assumed albedo of 0.04, and the most probable range of values for $N$ of $0.1 \leq N \leq 0.7$, where $N$ is the ratio of the constant nuclear flux to the total observed flux. See text. References: [1] Fay \& Wisniewski (1978), [2] Meech et al. (1998), [3] A’Hearn et al. (1995), [4] Weissman et al. (1999), [5] Lamy (1998), [6] Lamy et al. (1996), [7] Boehnhardt et al. (1999), [8] Lowry et al. (1999).

to have semi-axes of 3.8 and $2.9 \mathrm{~km}$ and a rotational period of 14-39 hours. The semi-minor axis value differs from our value of $2.4 \pm 0.1 \mathrm{~km}$ at the $4 \sigma$ level. A possible explanation is that the nucleus may be a triaxial ellipsoid and perhaps in a complex rotational state. This could account for both the discrepancy in radius and the range of possible rotational periods found. The radius of $22 \mathrm{P} / \mathrm{Kopff}$ was found to be $1.8 \pm 0.1 \mathrm{~km}$ at $5.11 \mathrm{AU}$ from the sun, in excellent agreement with Lamy et al. (1996) who observed the comet at $R_{\mathrm{h}}=0.57 \mathrm{AU}$ and used coma subtraction techniques to infer the nucleus size. $7 \mathrm{P} /$ Pons-Winnecke has a radius of $2.6 \pm 0.1 \mathrm{~km}$ derived from an $R$ magnitude of $22.2 \pm 0.1$. With an $m_{\text {coma }}$ value of $21.87 \pm 0.03$ it is possible that an unresolved coma could dominate the observed flux but we should note that at its heliocentric distance of $5.58 \mathrm{AU}$ the $\mathrm{H}_{2} \mathrm{O}$ sublimation rate is $\sim 5$ orders of magnitude less than that at $R_{\mathrm{h}} \leq 3 \mathrm{AU}$. Although a CO or $\mathrm{CO}_{2}$ driven coma may be present, we believe that the nucleus radius derived here for $7 \mathrm{P} /$ Pons-Winnecke represents the actual value. The nuclear radius upper limits of the six active comets presented in Table 3 need to be refined through future observations at greater $R_{\mathrm{h}}$. Indeed $74 \mathrm{P} /$ Smirnova-Chernykh was already observed in Paper I and possessed a $3 \sigma$ upper limit of $\leq 10.4 \mathrm{~km}$.

Unfortunately the main shortcoming with "snapshot" observations of this type, is that cometary nuclei do tend to be elongated to various degrees, and that a basic intrinsic property is their rotation. Restricting the discussion to the unresolved comets of Sect. 3.2, there is no known method to ascertain at which point in the rotational lightcurve our observations occurred, without further observations. Indeed, for any inactive comet, the variation in projected cross-sectional area of a rotating irregularly shaped nucleus may enable or prevent detection. For several of the unresolved comets previous observations have been made, as discussed above. In the case of 22P/Kopff and 49P/Arend-Rigaux our observations occurred when the projected cross-sectional area was near its mean value. In contrast, 9P/Temple 1 was observed at a low point in the phase curve, having a significantly smaller projected radius than that obtained by previous investigators. No information regarding nucleus size and rotation has yet been determined for $7 \mathrm{P} /$ Pons-Winnecke. Given that the average axis ratio of cometary nuclei based on measurements to date is $\sim 1.7$ (see Meech et al. 1998 and references therein), it is highly probable that the nucleus is indeed elongated. However, nuclei radii larger than $10 \mathrm{~km}$ in radius have been measured (Campins et al. 1987; Ó Ceallaigh et al. 1995; Meech et al. 1993), therefore our measurement of $2.6 \pm 0.1 \mathrm{~km}$ for the radius of $7 \mathrm{P} / \mathrm{Pons}^{-}$ Winnecke still separates it from this subset of larger nuclear radii even if one assumes an axial ratio of 2.6:1, i.e. one of the largest axial ratios accurately measured (Meech et al. 1993). We also note that if one applies a maximum previously observed axis ratio of 2.6:1 and a minimum measured albedo of 0.02 to the undetected comets of Sect. 3.1, their semi-major axes are all constrained to below $8 \mathrm{~km}$.

Ultimately, complete rotational lightcurves obtained through several filters, and measured at various epochs, for a large sample of comets is desirable. Unfortunately this kind of data is notoriously difficult to obtain. "Snapshot" observations have the disadvantage of sacrificing rotational information but have the advantage that a larger number of comets can be sampled at any given time. In this way, potential targets for future studies of nuclear properties can be identified. In fact, through Paper I and this work, several comets may be worthy of such programs. They include 69P/Taylor, 120P/Mueller 1, and 86P/Wild 3, all of which appeared stellar beyond $3 \mathrm{AU}$, are easily observable, and are extremely unlikely to be contaminated by an unresolved dust coma. Other potential targets are $81 \mathrm{P} /$ Wild 2, 43P/Wolf-Harrington, $79 \mathrm{P} / \mathrm{du}$ Toit-Hartley, and 7P/Pons-Winnecke. Although they too were stellar in appearance beyond $3 \mathrm{AU}$, the presence of a condensed unresolved dust coma cannot be ruled out. 
Table 5. Comparison of Af $\rho$ values with previous values listed in A'Hearn et al. (1995) [1] and Paper I [2]

\begin{tabular}{lccccc}
\hline Comet & Af $\rho[\mathrm{cm}]$ & $R_{\mathrm{h}}[\mathrm{AU}]$ & $\begin{array}{c}\text { Previous } A f \rho \\
\text { value }[\mathrm{cm}]\end{array}$ & $R_{\mathrm{h}}[\mathrm{AU}]$ & Ref. \\
\hline 6P/d'Arrest & $\leq 14.2$ & $5.63^{\dagger}$ & 21.4 & $1.43^{\ddagger}$ & 1 \\
9P/Tempel 1 & $\leq 11.2$ & $3.36^{\dagger}$ & $12.0 \pm 1.0$ & $3.51^{\dagger}$ & 2 \\
& & & 263.0 & $1.52-2.05^{\dagger}$ & 1 \\
22P/Kopff & $\leq 8.9$ & $5.11^{\ddagger}$ & 457.1 & $1.77^{\dagger}$ & 1 \\
65P/Gunn & $133.4 \pm 4.7$ & $4.43^{\ddagger}$ & 23.4 & $2.64^{\dagger}$ & 1 \\
74P/Smirnova-Chernykh & $298.9 \pm 11.3$ & $4.24^{\dagger}$ & $228.8 \pm 11.4$ & $4.61^{\ddagger}$ & 2 \\
& & & 602.6 & $3.56^{\star}$ & 1 \\
87P/Bus & $\leq 0.8$ & $4.32^{\dagger}$ & $7.4 \pm 0.1$ & $3.38^{\ddagger}$ & 2 \\
& & & 16.2 & $2.23^{\dagger}$ & 1 \\
& & & 251.2 & $3.15^{\ddagger}$ & 1 \\
103P/Hartley 2 & $49.3 \pm 4.8$ & $3.63^{\ddagger}$ & 245.5 & $1.04^{\ddagger}$ & 1 \\
\hline
\end{tabular}

$\dagger$ Pre-perihelion, $\star$ At perihelion, $\ddagger$ Post-perihelion.

Conversely, several comets have so far been observed to display significant levels of activity near aphelion, rendering them unsuitable for such nuclear studies. However, they are excellent candidates for studies of distant activity. Dust production levels for the active comets observed in this particular study shall now be discussed.

\subsection{Af $\rho$ measurements}

Of the ten detected comets, six display significant dust comae, which is a lower fraction of the total number of comets observed than in Paper I. As in Paper I a wide range of Af $\rho$ values was measured. The entire range extends from $\leq 0.8 \mathrm{~cm}$ for $87 \mathrm{P} /$ Bus to $298.9 \pm 11.3 \mathrm{~cm}$ for $74 \mathrm{P} /$ Smirnova-Chernykh.

Table 5 compares the present Af $\rho$ values with those listed in Paper I and A'Hearn et al. (1995) derived at different heliocentric distances. An Af $\rho$ value of $21.4 \mathrm{~cm}$ was previously measured for $6 \mathrm{P} / \mathrm{d}$ 'Arrest at a heliocentric distance of $1.43 \mathrm{AU}$. The present upper limit for this extremely low activity comet is $\leq 14.2 \mathrm{~cm}$ at $5.63 \mathrm{AU}$ from the sun. Therefore considering its low activity at $1.43 \mathrm{AU}$, the upper limit stated from these observations may be regarded as a grossly overestimated upper limit even if an unobservable dust coma was present.

In Paper I 9P/Tempel 1 showed signs of an extremely faint dust coma at a pre-perihelion distance of $3.51 \mathrm{AU}$ and an Af $\rho$ value of $12.0 \pm 1.0 \mathrm{~cm}$. However no dust coma was observed here at a similar pre-perihelion distance of $3.36 \mathrm{AU}$, with a firm Af $\rho$ upper limit of $\leq 11.2 \mathrm{~cm}$. The similarity between these two values measured at different epochs and similar pre-perihelion distances may be evidence that the faint coma detected through PSF subtraction in Paper I was due to low $S / N$ subsequently leading to slightly inaccurate PSF fitting. The bare nucleus was therefore probably observed, consistent with these observations taken with a much larger telescope, and with those taken with the HST (Lamy 1998).

In our observations $22 \mathrm{P} /$ Kopff was established as a point source detection at $R_{\mathrm{h}}=5.11$ AU. With a firm Af $\rho$ upper limit of $\leq 8.9 \mathrm{~cm}$ and an aphelion distance of 5.35 AU it would seem that this comet was inactive during its aphelion passage just prior to these observations. The relatively small Af $\rho$ value of $49.3 \pm 4.8 \mathrm{~cm}$ for $103 \mathrm{P} /$ Hartley 2 at $R_{\mathrm{h}}=3.63 \mathrm{AU}$ implies that this comet may also be inactive at aphelion (5.86 AU).

74P/Smirnova-Chernykh was observed in Paper I and was by far the most active comet in that dataset. Again this comet's dust production rate was much higher than the others with an $A f \rho$ value of $298.9 \pm 11.3 \mathrm{~cm}$. $65 \mathrm{P} / \mathrm{Gunn}$ also possessed significant activity with an $A f \rho$ value of $133.4 \pm 4.7 \mathrm{~cm}$ at $R_{\mathrm{h}}=4.43 \mathrm{AU}$. As such a large $A f \rho$ value was measured so close to aphelion $(4.74 \mathrm{AU})$ this comet is likely to be continuously outgassing throughout its orbit. However, $65 \mathrm{P} /$ Gunn was previously observed close to perihelion with an $A f \rho$ value of only $23.4 \mathrm{~cm}$ (A'Hearn et al. 1995). The reason for this huge increase in dust production with heliocentric distance is unknown, although one possibility is that our observations took place during an outburst. Unfortunately, another shortcoming of these observations is that we are unable to distinguish between our explanation and other possible interpretations without further observational data. However, our measurements do highlight the need for futher investigation of this comet to understand its behaviour at large heliocentric distances.

\section{Summary}

49P/Arend-Rigaux was observed close to perihelion at $R_{\mathrm{h}}=2.11$ AU. This comet-asteroid transition object was bright and stellar in appearance with the exception of a small linear feature emanating from the nucleus, for which the most likely explanation is a dust-jet emanating from an extremely small active portion of the nucleus surface. Its nucleus radius was measured to be $4.4 \pm 0.1 \mathrm{~km}$ (with an assumed albedo of 0.03 ), consistent with previous observations. Nuclear radius measurements and upper limits were measured for a further 21 comets in the heliocentric range $3.41 \mathrm{AU} \leq R_{\mathrm{h}} \leq 5.63 \mathrm{AU}$. Assuming an albedo of 0.04 , these values ranged from $1.8 \mathrm{~km} \leq r_{\mathrm{N}} \leq 2.6 \mathrm{~km}$ for the inactive comets, with upper limits for the active and undetected comets ranging from $0.6 \mathrm{~km} \leq r_{\mathrm{N}} \leq 12.7 \mathrm{~km}$. 
Even if one applies a maximum axis ratio of $2.6: 1$ and a minimum albedo of 0.02 to the undetected comets of Sect. 3.1, their semi-major axes are all constrained to below $8 \mathrm{~km}$. Our values are consistent with previous measurements with the exception of $9 \mathrm{P} /$ Tempel 1 , where an irregularly shaped nucleus in an excited rotational state may explain the discrepancy. Of the apparently inactive comets detected, only 7P/Pons-Winnecke shows evidence of a possible unresolved coma.

As in Paper I the activity of $74 \mathrm{P} /$ Smirnova-Chernykh dominated that of the other comets having more than twice the the dust production levels of $65 \mathrm{P} / \mathrm{Gunn}$, its nearest rival in terms of dust production. We identify $65 \mathrm{P} /$ Gunn as another comet strongly suspected of being continously outgassing throughout its entire 6.8 year orbit. Six of the ten detected comets displayed significant and diverse levels of coma activity with a correspondingly wide range of Af $\rho$ values, measured from $19.3 \mathrm{~cm} \leq A f \rho \leq 298.9 \mathrm{~cm}$. Af $\rho$ upper limits derived for the unresolved and undetected comets range from $0.8 \mathrm{~cm} \leq A f \rho \leq 20.0 \mathrm{~cm}$. Surface brightness profiles were also calculated for the outgassing comets and were comparable within measurement errors to steady state coma models.

Acknowledgements. The William Herschel Telescope is operated on the island of La Palma by the Royal Greenwich Observatory at the Spanish Observatorio del Roque de los Muchachos of the Instituto de Astrofisica de Canarias. Financial assistance from DENI is gratefully acknowledged.

\section{References}

A'Hearn, M. F., Schleicher, D. G., Feldman, P. D., Millis, R. L., \& Thompson, D. T. 1984, AJ, 89, 579

A'Hearn, M. F., Campins, H., Schleicher, D. G., \& Millis, R. L. 1989, ApJ, 347, 1155

A'Hearn, M. F., Millis, R. L., Schleicher, D. G., Osip, D. J., \& Birch, P. V. 1995, ICARUS, 118, 223

Birkett, C. M., Green, S. F., Zarnecki, J. C., \& Russell, K. S. 1987, MNRAS, 225, 285

Boehnhardt, H., \& Käufl, H. U. 1995, IAUC, 6274

Boehnhardt, H., Rainer, N., Birkle, K., \& Schwelm, G. 1999, A\&A, 341, 912

Brooke, T. Y., \& Knacke, R. F. 1986, ICARUS, 67, 80

Bus, S. J., Bowell, E., Harris, A. W., \& Hewitt, J. V. 1989, ICARUS, 77, 223
Campins, H., A'Hearn, M. F., \& McFadden, L. 1987, AJ, 316, 847

Cruikshank, D. P., \& Brown, R. H. 1983, ICARUS, 56, 377

Davis, L. E. 1989, A Reference Guide to the IRAF/APPHOT Package (NOAO, Tucson, Arizona)

Davis, L. E. 1994, A Reference Guide to the IRAF/DAOPHOT Package (NOAO, Tucson, Arizona)

Fay, T. D., \& Wisniewski, W. 1978, ICARUS, 34, 1

Festou, M. C., Rickman, H., \& West, R. M. 1993, A\&AR, 5, 37

Fitzsimmons, A., Dahlgren, M., Lagerkvist, C. I., Magnusson, P., \& Williams, I. P. 1994, A\&A, 282, 634

Jewitt, D. C., \& Danielson, G. E. 1984, ICARUS, 60, 435

Jewitt, D. C., \& Meech, K. J. 1987a, AJ, 93, 1542

Jewitt, D. C., \& Meech, K. J. 1987b, ApJ, 317, 992

Jewitt, D. C., \& Meech, K. J. 1988, AJ, 328, 974

Jewitt, D. C., \& Luu, J. 1989, AJ, 97, 1766

Keller, H. U., Delamere, W. A., \& Huebner, W. F., et al. 1987, A\&A, 187, 807

Lamy, P., \& Toth, I. 1995, A\&A, 293, L43

Lamy, P., A’Hearn, M. F., Toth, I., \& Weaver, H. A. 1996, Am. Astron. Soc. D.P.S. Meeting, 28

Lamy, P. 1998, IAU Circ., 7000

Landolt, A. U. 1992, AJ, 104, 340

Lowry, S. C., Fitzsimmons, A., Cartwright, I. M., \& Williams, I. P. 1999, A\&A, 349, 649

Massey, P. 1997, A User's Guide to CCD Reductions with IRAF (NOAO, Tucson, Arizona)

Meech, K. J., \& Jewitt, D. C. 1987, A\&A, 187, 585

Meech, K. J., Belton, M. J. S., Mueller, B. E. A., Dicksion, M. W., \& Li, H. R. 1993, AJ, 106, 1222

Meech, K. J., Knopp, G. P., \& Farnham, T. L. 1995, ICARUS, 116,46

Meech, K. J., \& Newburn, R. L. 1998, Am. Astron. Soc. D.P.S. Meeting, 30

Meech, K. J. 1998, in Asteroids, Comets, Meteors 1996, COSPAR Colloquium 10, in press

Millis, R. L., A’Hearn, M. F., \& Campins, H. 1988, ApJ, 324, 1194

Ó Ceallaigh, D. P., Fitzsimmons, A., \& Williams, I. P. 1995, A\&A, 297, L17

Pritchet, C., \& Kline, M. I. 1981, AJ, 86, 1859

Russell, H. N. 1916, ApJ, 43, 173

Scotti, J. V., \& Jedicke, R. 1994, IAU Circ., 6089

Sekanina, Z. 1974, The Study of Comets, ed. B. Donn, M. Mumma, W. Jackson, M. A'Hearn, \& R. Harrington, 537

Tokunaga, A. T., \& Hanner, M. S. 1985, ApJ, 296, L13

Veeder, G. J., Hanner, M. S., \& Tholen, D. J. 1987, AJ, 94, 169

Weissman, P., Doressoundiram, A., Hicks, M., et al. 1999, Am. Astron. Soc. D.P.S. Meeting, 31 